\title{
Analysis of Calcium Imaging Signals from the Honeybee Brain by Nonlinear Models
}

\author{
M. Stetter,*† H. Greve,* C. G. Galizia,ł and K. Obermayer* \\ *Department of Computer Science, Technische Universität Berlin, FR2-1, Franklinstrasse 28/29, D-10587 Berlin, Germany; \\ ‡Department of Neurobiology, Freie Universität Berlin, Königin-LuiseStrasse 28-30, D-14159 Berlin, Germany; and \\ †Corporate Technol ogy Department, ZT IK4, Siemens AG, D-81730 Munich, Germany
}

Received April 20, 2000; published online November 7, 2000

Recent $\mathrm{Ca}^{2+}$-imaging studies on the antennal lobe of the honeybee (Apis mellifera) have shown that olfactory stimuli evoke complex spatiotemporal changes of the intracellular $\mathrm{Ca}^{2+}$ concentration, in which stimulus-dependent subsets of glomeruli are highlighted. In this work we use nonlinear models for the quantitative identification of the spatial and temporal properties of the $\mathrm{Ca}^{2+}$-dependent fluorescence signal. This technique describes time series of the $\mathrm{Ca}^{2+}$ signal as a superposition of biophysically motivated model functions for photobleaching and $\mathrm{Ca}^{2+}$ dynamics and provides optimal estimates of their amplitudes (signal strengths) and time constants together with error measures. Using this method, we can reliably identify two different stimulus-dependent signal components. Their delays and rise times, $\delta_{\mathrm{c} 1}=(0.4 \pm 0.3) \mathrm{s}, \tau_{\mathrm{c} 1}=$ $(3.8 \pm 1.2) \mathrm{s}$ for the fast component and $\delta_{\mathrm{c} 2}=(2.4 \pm 0.6)$ $\mathrm{s}, \tau_{\mathrm{c} 2}=(10.3 \pm 3.2) \mathrm{s}$ for the slow component, are constant over space and across different odors and animals. In chronological experiments, the amplitude of the fast (slow) component often decreases (increases) with time. The pattern of the $\mathrm{Ca}^{2+}$ dynamics in space and time can be reliably described as a superposition of only two spatiotemporally separable patterns based on the fast and slow components. However, the distributions of both components over space turn out to differ from each other, and more work has to be done in order to specify their relationship with neuronal activity. 2001 Academic Press

\section{INTRODUCTION}

During the past decade, optical imaging of neuronal population activity has become an important experimental tool for the investigation of neuronal response properties and coding strategies. Optical imaging can be based on intrinsic signals (Grinvald et al., 1986; Bonhoeffer and Grinvald, 1996), voltage-sensitive dyes (Blasdel and Salama, 1986; Blasdel, 1992), or dyes sensitive to $\mathrm{Ca}^{2+}$ (Tsien, 1980; Poenie, 1992; Galizia et al., 1997) or other species. The latter technique, which is referred to as $\mathrm{Ca}^{2+}$ imaging, uses the facts that postsynaptic intracellular $\mathrm{Ca}^{2+}$ concentration often increases when the cells are activated and that a presynaptic calcium influx is necessary for transmitter re lease. Neural population activity is recorded by introducing a $\mathrm{Ca}^{2+}$-sensitive fluorescent dye into the intracellular neuronal space of the considered tissue, illuminating it, and recording the amount of fluorescent light over space and time during neuronal stimulation. The resulting change in the two-dimensional pattern of fluorescent light intensity is taken as a measure of the pattern of neuronal population activity. Recently, this technique has been successfully applied to characterize important aspects of neuronal olfactory coding in the antennal lobe of honeybees (J oerges et al., 1997; Galizia et al., 1998, 1999; Sachse et al., 1999).

However, the optical signal obtained in $\mathrm{Ca}^{2+}$-imaging experiments represents a composite signal (cf. also Fig. 2), the structure and the components of which have not yet been analyzed in depth. First, the signal contains at least one component that is due to photobleaching. Second, the intracellular $\mathrm{Ca}^{2+}$ concentration generally can change due to several mechanisms, not all of which are necessarily linked to neuronal activity. Third, autofluorescence may form another component of the fluorescence signal. This implies that the change of the intracellular $\mathrm{Ca}^{2+}$ concentration, which gives rise to the neuronal activity estimate, in general cannot be determined simply from the amplitude of the measured fluorescence composite signal, because this signal changes due to several causes. Hence, we face the issues of (i) the identification of the different signal components from the measured mixture and (ii) their separation and quantification.

Recently, blind source separation techniques, which are based on the assumptions of zero correlation functions or statistical independence between different signal sources, have been used to extract estimators for neural activity patterns from intrinsic signals. These algorithms use assumptions about the statistical struc- 
ture of the unknown sources, including statistical independence (Schießl et al., 1999) or spatial smoothness and vanishing cross-correlation functions (Stetter et al., 2000), for their separation.

In this work we complement these approaches by a model-based description of the imaging data, which is based on assumptions about the explicit time courses of signal sources instead of general assumptions about their statistical properties. We fit both individual time series and complete image stacks obtained from $\mathrm{Ca}^{2+}$ imaging experiments of the antennal lobe of honeybees (Apis mellifera) with nonlinear models. We first provide the mathematical framework of nonlinear modelbased approaches. Model functions are designed to reflect important aspects of prior knowledge about the signal behavior over time. We then demonstrate their potential: Nonlinear models can successfully explain the data, can separate different signal sources from each other, and can provide statistical parameter maps with improved signal-to-noise ratio thereby providing confidence intervals. These properties allow us to obtain the following key results: (i) In addition to photobleaching, the time series averaged over active regions of the antennal lobe contain both a fast and a slow component. (ii) For subsequent trials during an experiment, the amplitude of the fast component decreases over time, whereas the amplitude of the slow component increases. (iii) The spatial distributions of the amplitudes of both components in response to stimulation are not identical but remarkably similar to each other. These findings provide for the first time a consistent interpretation of the fluorescent signals, yet bring up the important issue of which neuronal processes underlie the two signal components we have observed.

The paper is organized as follows: Under Materials and Methods we describe the preparation and data collection procedures and explain our method of analysis. The analysis results which we have obtained for the $\mathrm{Ca}^{2+}$-imaging data from the antennal lobe are presented under Results and then discussed.

\section{MATERIALS AND METHODS}

\section{Animal Preparation, Staining, and Data Collection}

In vivo calcium recordings were performed as described elsewhere (Galizia et al., 1998). After capture from the hive, bees were quickly chilled for anesthetization and fixed in a plexiglass chamber. The head capsule was opened and the brain was floated in a solution of Calcium Green 1 or 2 AM (Molecular Probes, E ugene, OR; $50 \mu \mathrm{g}$ dye was first dissolved in 50 $\mu l$ Pluronic in DMSO and then diluted in $950 \mu \mathrm{l}$ Ringer, $130 \mathrm{mM} \mathrm{NaCl}, 6 \mathrm{mM} \mathrm{KCl}, 4 \mathrm{mM} \mathrm{MgCl}$, $5 \mathrm{mM}$ $\mathrm{CaCl}_{2}, 160 \mathrm{mM}$ sucrose, $25 \mathrm{mM}$ glucose, $10 \mathrm{mM}$ Hepes, $\mathrm{pH}$ 6.7, $500 \mathrm{mOsm})$. After $1 \mathrm{~h}$ staining, the brain was a

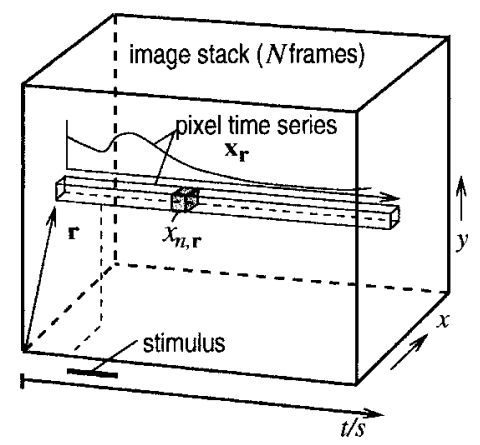

b
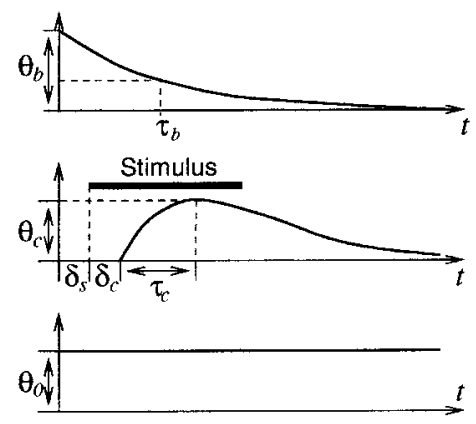

FIG. 1. (a) Data structure of the image stack gained from one trial. $\mathrm{N}$ successive frames are taken with a frequency of $2 \mathrm{~Hz}$ before, during, and after stimulation. Nonlinear models are applied to pixel time series $\mathbf{x}_{\mathrm{r}}$. (b) Model functions used. Top: Exponential function for photobleaching components. Middle: $\alpha$-function for stimulusevoked components. Bottom: Background component of fluorescence. $\theta_{b}, \theta_{c}, \theta_{0}$ are linear (amplitude) parameters, $\tau_{b}, \delta_{c}, \tau_{c}$ are nonlinear (curve shape) parameters.

rinsed in fresh Ringer and the recording chamber placed under the microscope with constant Ringer perfusion $(1 \mathrm{ml} / \mathrm{min})$ at room temperature $\left(22^{\circ} \mathrm{C}\right)$. At all stages, great care was taken not to wet the antennae.

I mages of a $240 \times 250-\mu \mathrm{m}$ region of the antennal lobe were taken with a 12-bit CCD camera (Photometrics $\mathrm{CH} 250 \mathrm{~A}$, Tucson, $\mathrm{AZ}$ ) at a rate of 2 images per second, with $240 \mathrm{~ms}$ exposure time per image. For every trial, either $\mathrm{N}=40$ or $\mathrm{N}=50$ images, corre sponding to 20 or $25 \mathrm{~s}$, were recorded, while an odor was applied to the antennae from $t=3 \mathrm{~s}$ until $t=5 \mathrm{~s}$ after the beginning of the image collection. Before storage, each image was spatially binned to a final size of $\mathrm{P}_{\mathrm{x}} \times \mathrm{P}_{\mathrm{y}}=49 \times 51$ pixels. After each trial, a 35- or 40-s recovery period without illumination preceded the next trial. One single experiment consisted of six trials with odor followed by one trial with plain air as stimulus (air trial). U sually, many experiments were carried out for a given animal.

Figure la illustrates the data format of an image stack as collected during one individual trial. The stack consists of a sequence of $\mathrm{N}$ images collected at times $t_{i}=0.5(i-1) s, i=1, \ldots, N$. The gray level of the pixel at location $\mathbf{r}=(\mathrm{x}, \mathrm{y}), 1 \leq \mathrm{x} \leq \mathrm{P}_{\mathrm{x}}, 1 \leq \mathrm{y} \leq \mathrm{P}_{\mathrm{y}}$ within the ith image is given by $x_{i, r}$ and reflects the amount of 
fluorescent light collected at pixel $\mathbf{r}$ and time $\mathrm{t}=$ $0.5(i-1)$. The $N \times P$ matrix, $P=P_{x} P_{y}$, of all pixel values $X_{i, r}$ describes an image stack and is denoted by $\mathbf{X}$. I ts col umn vectors $\mathbf{x}_{\mathbf{r}}=\left(\mathrm{x}_{1, \mathrm{r}}, \ldots, \mathrm{x}_{\mathrm{N}, \mathrm{r}}\right)^{\top}$ represent the time series of gray values for individual pixels $\mathbf{r}$ and are referred to as pixel time series. The superscript $T$ denotes the transpose of a vector.

\section{Nonlinear Models}

In this subsection we provide the mathematical framework for the statistical analysis of image stacks by nonlinear models. We follow the hypothesis that the change in fluorescence over time represents a composite signal. Because the relative changes measured are small compared to unity, we further assume that nonlinear interactions between the different signal components are negligible and describe the total signal as a linear superposition of different model functions, one for each signal component. Consequently, a given time series of signals, $\mathbf{x}=\left(\mathrm{x}_{1}, \ldots, \mathrm{x}_{\mathrm{N}}\right)^{\mathrm{T}}$, can be written as a weighted sum of model time series plus noise,

$$
\mathrm{x}_{\mathrm{i}}=\sum_{\mathrm{k}=1}^{\mathrm{K}} \mathrm{h}_{\mathrm{i}, \mathrm{k}}\left(\phi_{1}, \ldots, \phi_{\mathrm{L}}\right) \theta_{\mathrm{k}}+\mathrm{n}_{\mathrm{i}} \text {. }
$$

In Eq. (1), $h_{i, k}\left(\phi_{1}, \ldots, \phi_{L}\right)=h_{k}\left(\phi_{1}, \ldots, \phi_{L}, t_{i}\right)$ denotes a family of model functions for the kth signal component, sampled at time $t_{i}$ and parameterized by a set $\Phi=\left(\phi_{1}, \ldots, \phi_{\mathrm{L}}\right)^{\top}$ of parameters. Their values have to be estimated from the data. Because there is a nonlinear relationship between $\Phi$ and the data, these parameters are referred to as nonlinear parameters, and our approach Eq. (1) is referred to as a nonlinear model of the data. The components of $\Phi$ parameterize the curve shape of a given model function. The set $\Theta=\left(\theta_{1}, \ldots\right.$, $\theta_{K}$ ) specifies the linear mixing coefficients of the signal components and they are referred to as linear parameters. They provide the amplitude or signal strength of every component within the time series and must also be estimated from the data. The components of the vector $\mathbf{n}$ describe the noise. In the following we assume the noise to be Gaussian, $\mathrm{n}_{\mathrm{i}}=\mathrm{N}\left(0, \sigma_{\mathrm{n}}^{2}\right)$, with zero mean and $\sigma_{\mathrm{n}}^{2}$ variance. This assumption seems well justified, because noise in the data is dominated by photon shot noise which follows a Poisson distribution. Because the gray level of each pixel is generated by many photons and because detection events at different pixels and times are independent, the Poisson distribution can be well approximated by a Gaussian distribution as provided above. If we combine the set of model time series $\mathbf{h}_{\mathrm{k}}$ to form the column vectors of the design matrix $\mathbf{H}$ with $\mathbf{H}_{\mathrm{i}, \mathrm{k}}=\mathrm{h}_{\mathrm{i}, \mathrm{k}}$, we can write Eq. (1) in matrix form:

$$
\mathbf{x}=\mathbf{H}(\Phi) \Theta+\mathbf{n}
$$

Parameters are estimated using the principle of maximum likelihood:

$$
\begin{aligned}
\mathrm{p}(\mathbf{x} \mid \Theta, \Phi)= & \frac{1}{\left(2 \pi \sigma_{\mathrm{n}}^{2}\right)^{\mathrm{N} / 2}} \exp \left(-\frac{\mathbf{n}^{\top} \mathbf{n}}{2 \sigma_{\mathrm{n}}^{2}}\right) \\
= & \frac{1}{\left(2 \pi \sigma_{\mathrm{n}}^{2}\right)^{\mathrm{N} / 2}} \exp \left(-\frac{1}{2 \sigma_{\mathrm{n}}^{2}}(\mathbf{x}-\mathbf{H}(\Phi) \Theta)^{\top}\right. \\
& \times(\mathbf{x}-\mathbf{H}(\Phi) \Theta))=\max
\end{aligned}
$$

F or a fixed but arbitrary set of nonlinear parameters, $\Phi$, finding the optimal linear parameters $\Theta_{\text {opt }}(\Phi)$ reduces to a linear problem, which can be solved analytically (Kay, 1993):

$$
\Theta_{\text {Opt }}(\Phi)=\left(\mathbf{H}^{\top}(\Phi) \mathbf{H}(\Phi)\right)^{-1} \mathbf{H}^{\top}(\Phi) \mathbf{x} .
$$

The residual, i.e., the vector of differences between the data and the model is then given by

$$
\begin{aligned}
& \mathbf{R}(\Phi)=\mathbf{x}-\mathbf{H}(\Phi) \Theta_{\mathrm{Opt}} \\
&=\left(\mathbf{I}-\mathbf{H}(\Phi)\left(\mathbf{H}^{\top}(\Phi) \mathbf{H}(\Phi)\right)^{-1} \mathbf{H}^{\top}(\Phi)\right) \mathbf{x} \\
& \mathbf{X}=: \mathbf{P}(\Phi) \mathbf{x}
\end{aligned}
$$

with I being the $\mathrm{N} \times \mathrm{N}$ unit matrix. The optimal nonlinear parameters $\Phi_{\text {opt }}$ can be obtained by minimizing the cost function,

$$
E(\Phi)=\sum_{i=1}^{N} R_{i}^{2}(\Phi),
$$

and the optimal linear parameters result from Eq. (4) as $\Theta_{\text {opt }}\left(\Phi_{\text {opt }}\right)$.

Once the optimal parameters are known, the noise variance $\sigma_{\mathrm{n}}^{2}$ can be estimated as

$$
\hat{\sigma}_{\mathrm{n}}^{2}=\frac{\mathbf{R}^{\top}\left(\Phi_{\text {opt }}\right) \mathbf{R}\left(\Phi_{\text {opt }}\right)}{\operatorname{tr}\left(\mathbf{P}\left(\Phi_{\text {opt }}\right)\right)},
$$

where the trace operator tr of a matrix is the sum of its diagonal elements. The estimated signal-to-noise ratio for the kth amplitude parameter, which we refer to as its Z score, becomes

$$
Z_{k}=\frac{\left|\hat{\theta}_{k}\right|}{\hat{\sigma}_{\mathrm{k}}}=\frac{\left|\hat{\theta}_{\mathrm{k}}\right|}{\hat{\sigma}_{\mathrm{n}} \sqrt{\left[\left(\mathbf{H}^{\top}\left(\Phi_{\mathrm{Opt}}\right) \mathbf{H}\left(\Phi_{\mathrm{Opt}}\right)\right)^{-1}\right]_{\mathrm{k}, \mathrm{k}}}} .
$$

Estimates can be carried out pixel wise for all pixel time series of the image stack, $\mathbf{x}_{\mathrm{r}}$. In this case Eqs. (2)-(8) 
yield statistical parameter maps (SPM) $\Phi_{\text {opt, } r}, \Theta_{\text {Opt, } r}$, and $\mathbf{Z}_{\mathbf{r}}$. In particular, the SPM $\theta_{\text {Opt,k,r }}$ specifies how the strength of the kth signal component is distributed over space.

\section{Model Functions}

For application to $\mathrm{Ca}^{2+}$-imaging data, model functions $h_{k}(\Phi, t)$ were chosen as follows (Fig. 1b). First, the fluorescence signal is subject to photobleaching, which might be caused by one or several different mechanisms. Photobleaching is described by an exponential function,

$$
\mathrm{h}_{\mathrm{b}}\left(\tau_{\mathrm{b}, \mathrm{m}}, \mathrm{t}\right)=\exp \left(-\frac{\mathrm{t}}{\tau_{\mathrm{b}, \mathrm{m}}}\right)
$$

(Fig. 1b, top), where $m$ refers to a particular mechanism and $\tau_{b, m}$ is the corresponding time constant. Stimulus-evoked changes in fluorescence contain both a $\mathrm{Ca}^{2+}$-release mechanism from internal stores or the influx of extracellular calcium and a subsequent buffer mechanism (Tsien and Tsien, 1990; Naraghi and Neher, 1997; Xu et al., 1997). When neurons become active during a small period of time, a first-order calciumrelease mechanism will increase the concentration $\mathrm{c}(\mathrm{t})$ of free $\mathrm{Ca}^{2+}$ according to $\mathrm{c}(\mathrm{t})=\mathrm{c}(0)+(\mathrm{c}(\infty)-\mathrm{c}(0))(1-$ $\exp (-t / \tau)$ ) (with some time constant $\tau$ ), which can be well approximated by a linear function during the early phase. At the same time, a first-order buffer mechanism tries to restore the resting level by an exponential decay. Hence, if we assume first-order reaction kinetics to hold for the $\mathrm{Ca}^{2+}$-release and buffer mechanisms, the $\alpha$ function,

$$
\mathrm{h}_{\mathrm{c}}\left(\delta_{\mathrm{c}, \mathrm{m}}, \tau_{\mathrm{c}, \mathrm{m}}, \mathrm{t}\right)=\mathrm{h}_{0} \frac{\mathrm{t}-\delta_{\mathrm{c}, \mathrm{m}}-\delta_{\mathrm{s}}}{\tau_{\mathrm{c}, \mathrm{m}}} \exp \left(-\frac{\mathrm{t}-\delta_{\mathrm{c}, \mathrm{m}}-\delta_{\mathrm{s}}}{\tau_{\mathrm{c}, \mathrm{m}}}\right)
$$

(Fig. 1b, center), with its initial linear increase and its late exponential decay, represents a good phenomenological description of a stimulus-evoked component. In Eq. (10), $\delta_{\mathrm{s}}$ denotes the time of the stimulus onset, $\delta_{c, m}$ is the response latency, and $\tau_{c, m}$ denotes the rise time of the response for the mth stimulus-related component. $\mathrm{h}_{0}$ is a constant which normalizes the function to a maximal value of 1 . Finally, a constant function describes the background fluorescence (Fig. 1b, bottom).

For example, a nonlinear model with one bleaching component and two stimulus-related components is described by the parameter sets $\Theta=\left(\theta_{0}, \theta_{\mathrm{b}}, \theta_{\mathrm{c} 1}, \theta_{\mathrm{c} 2}\right)$, where $\theta_{0}$ is the amplitude of the background fluorescence, and $\Phi=\left(\tau_{\mathrm{b}}, \delta_{\mathrm{c} 1}, \tau_{\mathrm{c} 1}, \delta_{\mathrm{c} 2}, \tau_{\mathrm{c} 2}\right)$.

For the model functions and parameterizations used, the cost function turned out to be relatively smooth and the optimization by standard numerical gradient de- scent quickly converged to a unique result. This indicated that the optimization process was not disturbed by local minima. I nitial values of nonlinear parameters were set as estimated by visual inspection from the $\Delta \mathrm{F} / \mathrm{F}$ image to $\delta_{\mathrm{c} 1}=0.25 \mathrm{~s} ; \delta_{\mathrm{c} 2}=2 \mathrm{~s} ; \tau_{\mathrm{c} 1}^{-1}=0.10 \mathrm{~s} ; \tau_{\mathrm{c} 2}^{-1}=$ $0.05 \mathrm{~s}$. The gradient was calculated numerically using step sizes of $(0.01,0.01,0.0004,0.0002)$ for the four parameters. The step size for the parameter update was adapted as follows: If the gradient along a direction changed sign in two subsequent steps, the step size was decreased by a factor of 2 ; if its sign was preserved for three subsequent update steps, the step size was multiplied by a factor of 1.25. Programs were implemented in Matlab on a Sun Ultra 10 workstation. Computation time was approximately $1 \mathrm{~s}$ per pixel time series.

\section{RESULTS \\ Identification of Signal Components from Mean Time Series}

For the identification of different components of fluorescence, nonlinear model fits were first applied to mean time series taken over an active region, i.e., over a region where the fluorescence increased in response to an odor. The approximate extensions of active regions were determined prior to the analysis from the $\Delta \mathrm{F} / \mathrm{F}$ image (J oerges et al., 1997), which we obtained as follows:

1. Use a stimulus-trial $\mathbf{X}_{i, r}$.

2. Calculate mean fluorescence before stimulation: $\mathbf{F}_{\mathbf{r}}=\frac{1}{6} \sum_{\mathrm{i}=1}^{6}\left(\mathbf{X}_{\mathrm{i}, \mathrm{r}}\right)$.

3. Calculate mean fluorescence during maximal response: $\mathbf{F}_{1, \mathrm{r}}=\frac{1}{4} \sum_{\mathrm{i}=10}^{13}\left(\mathbf{X}_{\mathrm{i}, \mathrm{r}}\right)$.

4. $(\Delta \mathrm{F} / \mathrm{F})_{\mathbf{r}}=\left(\mathbf{F}_{1, \mathbf{r}}-\mathbf{F}_{\mathbf{r}}\right) / \mathbf{F}_{\mathbf{r}}$.

Figure 2a shows the color-coded $\Delta F / F$ image of an antennal lobe for the sum over seven trials under stimulation with hexanol, showing approximately $1 \%$ change in fluorescence in the central lower part of the image. This region corresponds to a set of glomeruli (the functional units of the antennal lobe), which have been driven by the presentation of hexanol. $\Delta \mathrm{F} / \mathrm{F}$ images represent the common way of analyzing $\mathrm{Ca}^{2+}$ imaging data. For the rest of the paper we aim at a more detailed characterization of the fluorescence signal in space and time.

The solid lines with black circles in Figs. $2 b$ and $2 c$ represent the mean time series obtained by averaging over the pixel time series within the region of interest marked in Fig. 2a. The negative slope prior to stimulation indicates photobleaching and the increase starting from $t=3 \mathrm{~s}$ corresponds to a stimulated response. The dashed line in Fig. 2b shows the best fit, if one bleaching component and one stimulus-evoked component are used. The photobleaching component is over- 

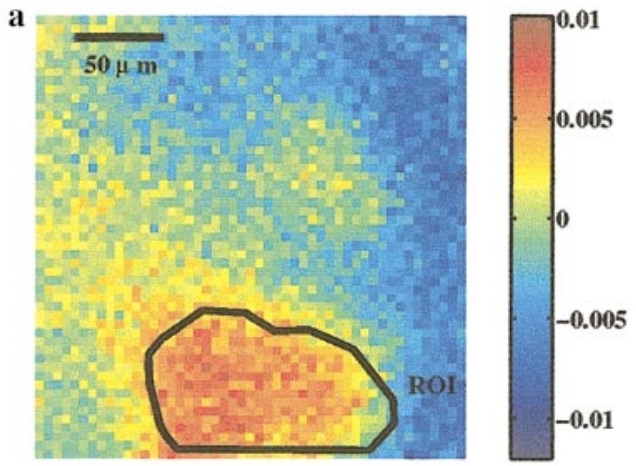

b

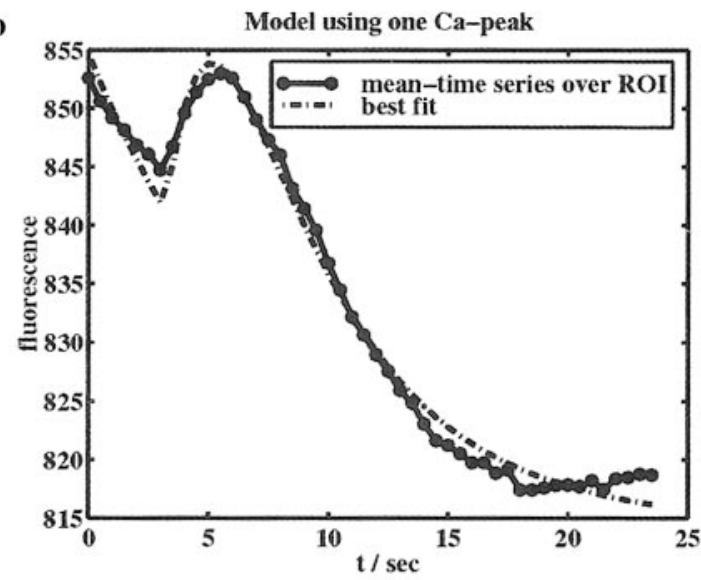

c

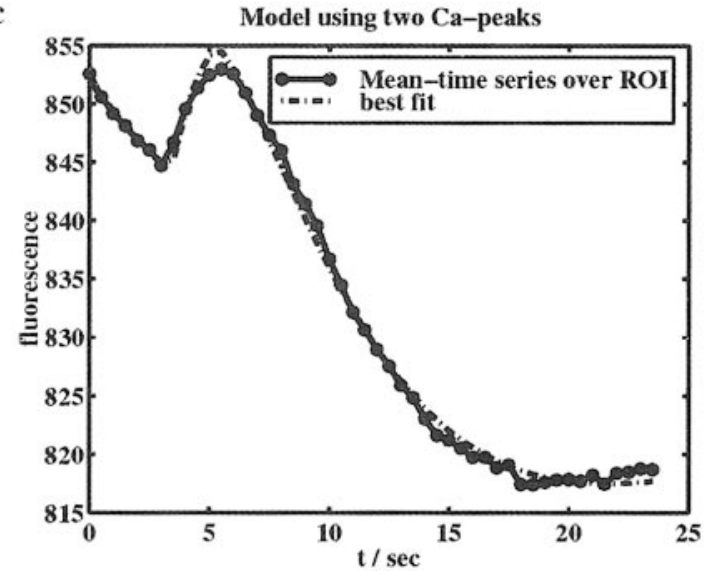

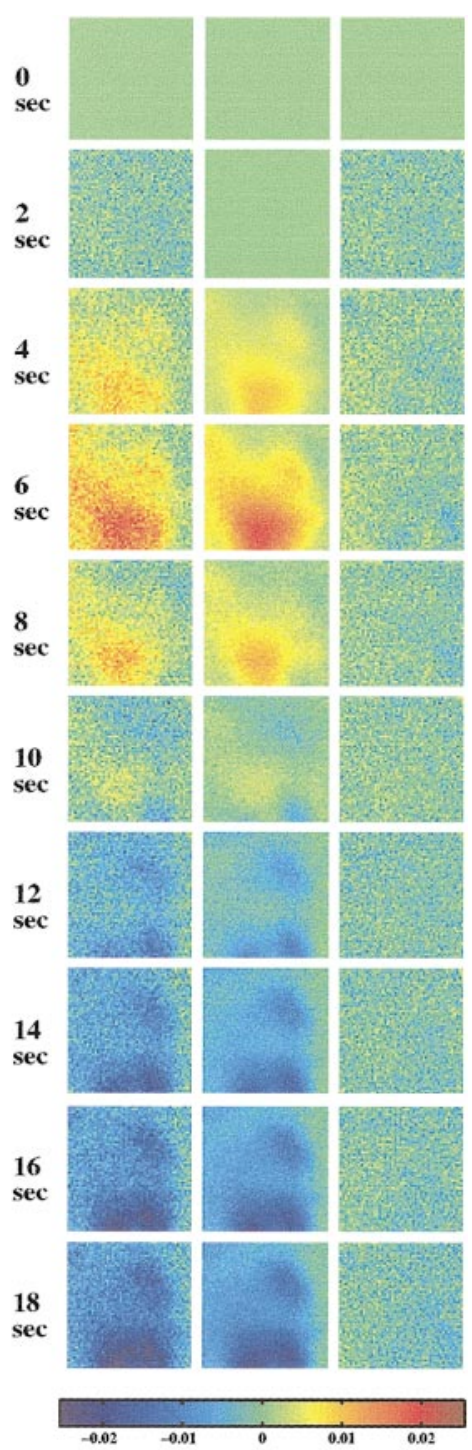

5

FIG. 2. Nonlinear model analysis of a mean time series. (a) Color-coded distribution of the relative change in fluorescence, $\Delta F / F$, due to stimulation. The black line circumscribes the region used for averaging the time series to form the mean time series. (b) Mean time series (black circles) and the best nonlinear fit using one photobleaching and one stimulus-evoked component, and (c) best fit for one bleaching and two stimulus-evoked components. Two stimulus-related components are needed for a correct description.

FIG. 5. Spatiotemporal fit of a whole image stack. Left column: Same image stack as analyzed in Fig. 2 after model-based correction for photobleaching (for further explanation see text). Only every fourth frame is shown. Middle column: The best pixel-wise fit of the data using the two signal components as determined from mean time series. Right column: Residual image stack (model subtracted from data). The residual contains little structure, indicating that the model successfully extracts all the relevant information from the data.

estimated, as can be seen from its high slope in the early phase. This poor performance is caused by a negative undershoot in the late phase of the data. Figure 2c (dashed) shows the best fit for one bleaching and two different stimulus-evoked components. The second component can account for the undershoot and the model provides a good fit. Use of more than two stimulus-evoked components resulted in an underdetermined system with a partially flat cost function, and likewise did the use of more than one photobleaching component (also in combination with one stimulusevoked component). We summarize that for this par- 

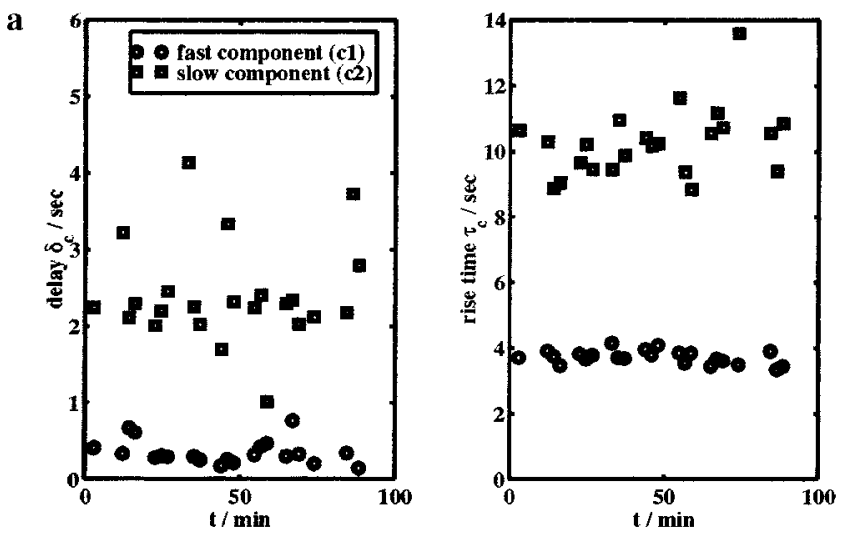

$\mathbf{b}$
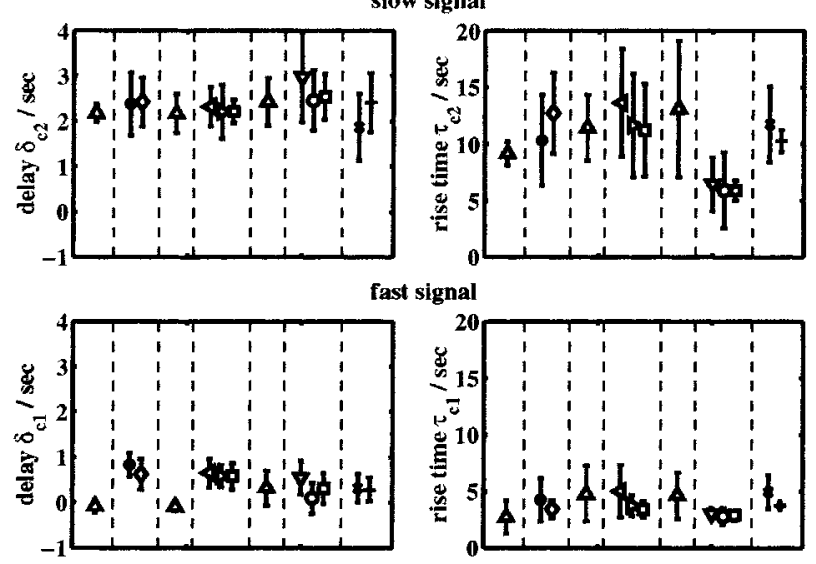

ast signal

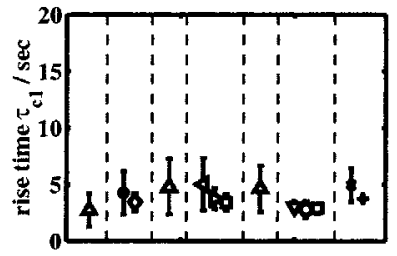

FIG. 3. (a) Delay (left) and rise time (right) parameters for mean time series from 23 consecutive trials on the same animal (stimulus: hexanol). All parameters are remarkably constant over time and between trials. Mean values: $\delta_{\mathrm{c} 1}=0.37 \mathrm{~s}, \tau_{\mathrm{c} 1}=3.85 \mathrm{~s}, \delta_{\mathrm{c} 2}=2.37 \mathrm{~s}$, $\tau_{\mathrm{c} 2}=9.54 \mathrm{~s}$. For all parameters, linear regression yielded absolute slopes of less than $0.015 \mathrm{~s}$ per minute duration of the experiment. (b) Mean delays and rise-time parameters for different odors and odor combinations and for seven different animals. Vertical dashed lines separate different animals, error bars mark the standard deviations of the series as shown in (a). All nonlinear parameters are very constant over time, between odors, and between animals. Symbols: $(\triangle)$ hexanol as part of mixed odor; $(\bullet)$ isoamylacetate + hexanal mix; $(\diamond)$ citral + hexanol mix; $(\triangleleft)$ limonene, hexanol (mean of individual stimulations); $(\triangleright)$ limonene + hexanol mix; $(\square)$ octanol; $(\nabla)$ limonene; (○) clove oil; ( $\square$ ) lavender; $(\times)$ isoamylacetate; (+) hexanol;

ticular data set, one bleaching and two different stimulus-evoked components, a fast positive and a slow negative one, are necessary and sufficient for the description of the change in fluorescence over time. We will henceforth refer to the fast and slow stimulusevoked components as signal components or signals.

The model analysis using the one-bleaching two-signals model was repeated on many mean time series, which we obtained from single trials for different odors and from different animals. Figure 3a shows the nonlinear parameters for a sequence of 23 subsequent trials for the same animal as in Fig. 2 (other odors were tested intermittently). It demonstrates that the delays and rise times of the two signal components remain remarkably constant over time and between trials. The analysis was carried out on 13 sequences of trials as shown in Fig. 3a, taken from seven different animals. Mean values and standard deviations were determined for each sequence and are summarized in Fig. 3b. The curve shape parameters do not vary strongly between different odors and different animals. Finally we analyzed sequences of trials both under application of the sodium channel blocker TTX and under a $\mathrm{Ca}^{2+}$-free environment and found that both components are reversibly blocked by these procedures (data not shown). Also, imaging on preparations without staining did not result in a significant presence of signal components. These results demonstrate the existence of two different stimulus-related components in the fluorescence signal with well-defined and well-separable dynamical parameters. Their mean delays and rise times over all 210 trials analyzed were determined as $\delta_{\mathrm{c} 1}=(0.4 \pm$ $0.3) \mathrm{s}, \tau_{\mathrm{c} 1}=(3.8 \pm 1.2) \mathrm{s}$ for the fast component and $\delta_{\mathrm{c} 2}=(2.4 \pm 0.6) \mathrm{s}, \tau_{\mathrm{c} 2}=(10.3 \pm 3.2) \mathrm{s}$ for the slow component. Errors include variations between animals. Because of this constancy, the nonlinear parameters were kept constant at their mean values for the following considerations.

The top row of plots in Fig. 4 shows the relative amplitudes of the fast and slow signal components, $\theta_{\text {opt },} / \theta_{\text {opt }, 0}$, for the same sequence of trials as in Fig. 3a; the plots below contain the corresponding $Z$ scores. There are three major observations: (i) The large $Z$ scores indicate that the presence of both components is highly significant. (ii) Both relative changes are comparable in absolute size, and the peak changes are estimated to be approximately three times as large as
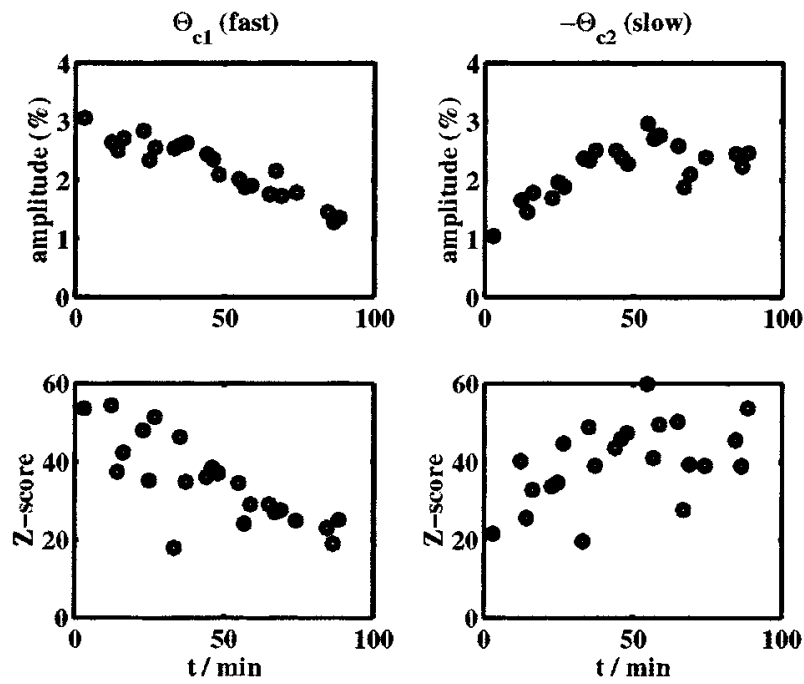

FIG. 4. Chronological behavior of the relative changes in amplitude for the fast (top left) and slow (top right) signal components for 23 subsequent and identical trials (stimulus: hexanol). The fast component decays while the slow component tends to increase in strength over time. Bottom plots: Corresponding Z scores of the amplitudes above. The presence of both signals is highly significant. 
from the simple $\Delta F / F$ image. This is due to the fact that our analysis method accounts for the full signal, including its curve shape, and that both components overlap and thereby partially cancel each other in the measured mean time series. (iii) The fast component decreases over time (rate: $-0.011 / h$ ), whereas the slow components show a tendency to increase until a saturation is reached approximately $1 \mathrm{~h}$ after the beginning of the experiment (rate: $0.019 / \mathrm{h}$ ). This behavior is observed in about $50 \%$ of the sequences analyzed, in the remaining trials there was a much weaker or even no change in amplitude over time.

\section{Analysis of Spatial Distributions}

In this subsection we present results obtained from the pixelwise application of nonlinear models to all pixel time series of whole image stacks. Because individual pixel time series were more noisy than mean time series, results improved, if we first corrected for photobleaching and afterward fitted a model using only the background component and the two signal components. For the correction, we fitted a nonlinear model to the air trial of each experiment and subtracted the best model from the corresponding stimulus trials. This procedure avoids adding noise from the air trial to the stimulus trial, which would happen with simple subtraction. After correction, each pixel time series of a trial was fitted using a background component and the model functions of the fast and the slow components as determined from the corresponding mean time series. Note that the nonlinear curve shape parameters were kept fixed for each image stack. Therefore, only three amplitude parameters had to be fitted for each time series.

Figure 5 shows the best fit as described above for the same data set as used in Fig. 2. The left column shows every fourth frame of the bleach-corrected original image stack, the middle column the best model fit, and the right column the residual, which is the difference between the data and the model. First, the residual contains little structure, which indicates that all the relevant structure in the data is successfully captured by the model. Because the curve shape of the model functions is kept constant, the whole spatiotemporal signal can be explained by the presence of only two signal components. In other words, the temporal dynamics of the signal components are not only constant between odors and animals, but are also constant over space within the same animal. Second, it becomes visible that the spatial patterns of the fast and slow components are different from each other. This property is demonstrated more strikingly in Figs. 6 and 7.

In Fig. 6, the statistical parameter maps (top row) and the corresponding $Z$ scores (middle row) are shown for the fit of Fig. 5. The signals we extract are much less noisy than when only $\Delta \mathrm{F} / \mathrm{F}$ is used, and by the $\mathrm{Z}$ score we can quantify our confidence in them. Note that these maps result from only a temporal analysis, i.e., no spatial processing such as smoothing has been applied. The bottom of the figure is the difference be tween the strengths of the fast and slow components. It is nonzero, in this case amounts to approximately $30 \%$ of the signal components, and is spatially modulated. We conclude that the spatial distributions of the fast and the slow components are similar, but can differ from each other in the details of their structure.

This effect is demonstrated again in Fig. 7, which shows the spatial distributions of the fast and slow responses for a different animal and for three different stimulus conditions. In all cases, the fast and the slow components for a given odor are more similar to each other than to any component of another odor. However, they are not identical to each other. The fast and slow components usually show the same pattern of glomeruli; however, the intensity distributions differ from each other. Sometimes, this difference is large for an individual glomerulus, which then appears only in one of the components (e.g., top left and top right, right edge). The second aspect shown in Fig. 7 is the approximate linearity of the fluorescence signals. From top to bottom, the first three rows show responses to limonene, hexanol, and a mixture of both odors. The maps in the fourth row are obtained by a linear superposition of the maps for stimulation with the pure odors. If the fluorescence signal behaved linearly, the mixture of odors would evoke the same response as the superposition of the responses to the pure odors. A comparison of the third and fourth rows from the top with each other demonstrates an expressed similarity of the spatial patterns for both cases, which corroborates the linearity assumption. However, the total strength is somewhat weaker in the response to mixed odors than expected by a linear behavior, which hints toward the presence of lateral inhibition in the antennal lobe. This difference in strength is demonstrated by the two bottom plots, which display the difference between the response to odor mix and the superposition.

\section{DISCUSSION}

In the present work we fitted sets of nonlinear model functions to the time courses of fluorescence signals from $\mathrm{Ca}^{2+}$-imaging experiments. Using this technique, we were able to identify two well-defined and welldistinguishable dynamic components of the stimulusrelated fluorescence signal- one signal corresponds to a relatively fast increase in local fluorescence, whereas the second component reflects a slower decrease below background fluorescence. The temporal dynamics of both components are very constant over space as well as over different trials, odors, and animals. However, the spatial amplitude distributions of both components can differ from each other. 

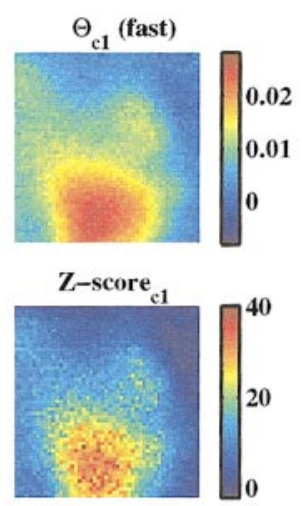

6
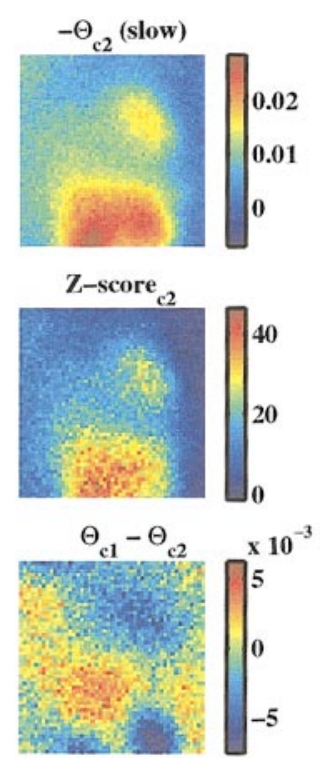

Fast

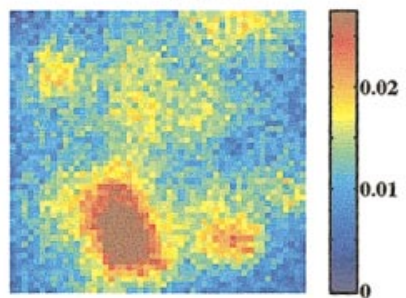

limonene
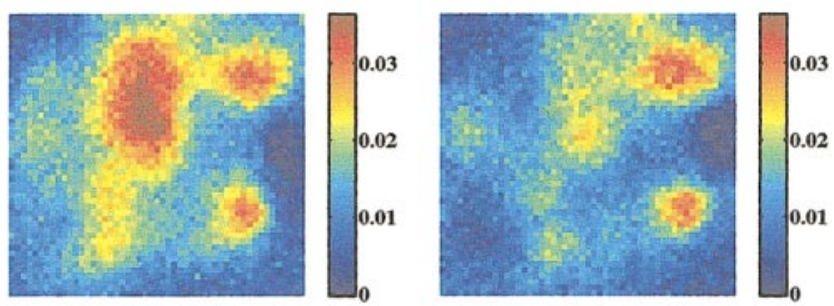

hexanol
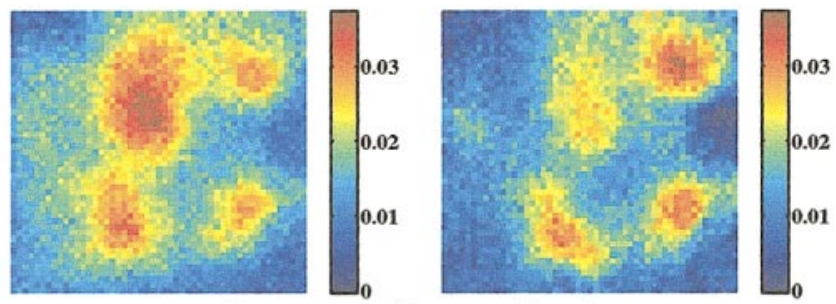

limonene/hexanol mix
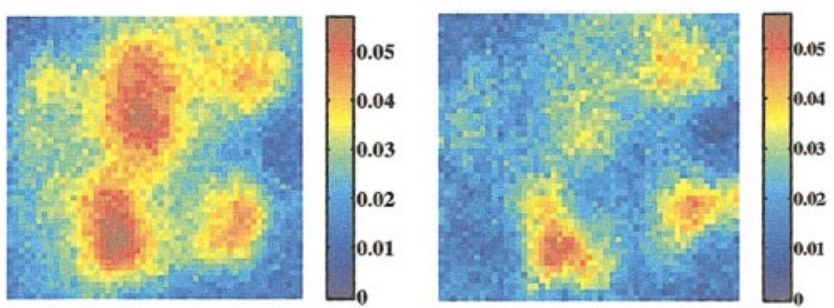

superposition limonene/hexanol
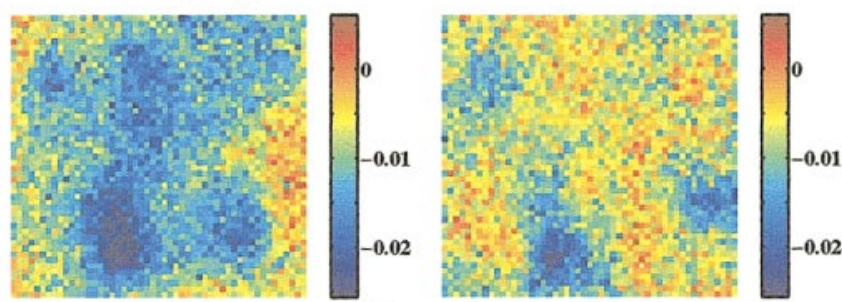

difference mix - superposition

FIG. 6. Top: Statistical parameter maps of the fast (left) and slow (right) components for the fit shown in Fig. 5. Both maps are much less noisy than the corresponding $\Delta \mathrm{F} / \mathrm{F}$ image (cf. Fig. 2a). Middle: Z scores of both signal components over space. Bottom: The difference between the fast and the slow component amounts to approximately $30 \%$ of each component. I ts spatial modulation indicates that the fast and slow components are differently distributed over space.

FIG. 7. Different examples for the spatial maps of fast (left) and slow (right) components. The respective patterns for stimulation with limonene (top row), stimulation with hexanol (second row), and stimulation with a mixture of both odors (third row) are similar but not identical for the fast and slow components. Fourth row: Linear superposition of responses to pure odors and (bottom row) the difference between the response to the mix and the superposition. Both mix and superposition patterns are similar to each other in space; however, the response to the mix is slightly smaller than expected by a linear superposition. 
The goal of $\mathrm{Ca}^{2+}$ imaging in this system is to infer the spatial or spatiotemporal patterns of neuronal population activity in response to olfactory stimulation, exploiting the relationship between neuronal activity and the amount of intracellular $\mathrm{Ca}^{2+}$. This $\mathrm{Ca}^{2+}$ level is monitored by measuring the change in fluorescence caused by a $\mathrm{Ca}^{2+}$-sensitive dye. Our identification of two different components of stimulus-related fluorescence with slightly different spatial distributions raises the important issue of what is the relationship between each of the components and neuronal activity. Possibly the different components reflect different celIular mechanisms of $\mathrm{Ca}^{2+}$ supply, which in turn might be related to different stages of olfactory coding. For example, the fast and slow components may reflect successive stages of olfactory processing in the antennal lobe. In this case, the differences of the spatial pattern of the two components would indicate that odors elicit not just a fixed set of activated glomeruli, but rather a slow sequence, with some glomeruli being activated with a delay. More biophysical work has to be done in order to specify the origin of each component as well as their relationship with neural activity. We hope that our characterization of the behavior of the responses over time and space can help address these questions.

In the cal cium measurements analyzed in this paper, the dye was Calcium Green AM, which was applied to the animal in vivo. In this situation, the dye is taken up by all cells, i.e., olfactory receptor cells, interneurons, and possibly glial cells. The relative concentration in these cells, and the contribution of these cells to the signal, is unknown. Furthermore, AM dyes have the tendency to internalize in intracellular calcium stores, so that signal contribution from intracellular store cannot be excluded. It appears all the more remarkable that our approach shows that with just two signal functions the measurements can be modeled.

Nevertheless, some speculations about the origin of both signals should be discussed. First, both signal components can be reversibly removed either by application of TTX or by removal of free extracellular $\mathrm{Ca}^{2+}$, both of which block neural activity in the antennal lobe. Also, both components are statistically insignificant in most air trials (in some air trials, weak stimulus-related components were observed, but we assigned those to residual odor impurities in the air used and excluded the data sets from the analysis). The first and the third observations indicate that the fluorescence signals are coupled to neuronal activity, whereas the second test hints toward a coupling either on activity or on a $\mathrm{Ca}^{2+}$ influx. Alternatively, one might argue that autofluorescence contributes to the measured change in fluorescence and could provide one of the signal components. However, in experiments carried out without staining, no significant fluorescence signals have been observed. Thus, neuronal activity and the $\mathrm{Ca}^{2+}$-sensitive fluorescent dye are necessary for the generation of both components.

The origin of the two components is still unclear, as is the reason for them behaving differently over space. For example, the slow signal component always had a negative amplitude, which apparently reflects a strong decrease in intracellular $\mathrm{Ca}^{2+}$ concentration. However, $\mathrm{Ca}^{2+}$ ions are very effectively buffered in the intracellular space, resulting in a very low concentration in the range of $100 \mathrm{nM}$ (Bygrave and Benedetti, 1996). Therefore, even a complete removal of free $\mathrm{Ca}^{2+}$ could not account for the strong undershoot observed. One alternative possibility of its origin could be a compartmentalization of the fluorescent dye into the endoplasmatic reticulum (ER), which operates as a $\mathrm{Ca}^{2+}$ reservoir (Virgilio et al., 1990; Slayman and Moussatos, 1994; Kao, 1994). This interpretation is corroborated by the increase of the slow component over time, which reflects the increase of compartmentalized dye. Pharmacological studies have to be undertaken to prove this hypothesis, whereby the second component would re flect $a \mathrm{Ca}^{2+}$ release out of the ER (Bygrave and Benedetti, 1996). Our nonlinear model would then allow us to segregate the contribution of extracellular calcium influx (first component) and intracellular calcium release (slow component, which would thus reflect a calcium decrease in the stores) in a single measurement. Alternatively, the signal could be composed from different origins: For example, the two signal components might be generated by different mechanisms in different cell types (Verkhratsky and Kettenmann, 1996).

The present method characterizes fluorescence signals with much higher accuracy than $\Delta F / F$ images and additionally provides confidence levels. By use of these properties it becomes possible to address new aspects of neuronal encoding of olfactory stimuli. For example, the strength and the type of nonlinearities in the code of composite stimuli (J oerges et al., 1997) can be quantified and tested for significance. Also, we have started to work on a method for identifying the positions of individual glomeruli directly from sets of $\mathrm{Ca}^{2+}$-imaging experiments by use of the $Z$ score. Based on those methods, it will become possible to quantitatively characterize the vector coding strategy, by which olfactory stimuli are represented in the antennal lobe of honeybees (Galizia et al., 1999). Finally, the mathematical method itself is not confined to a given imaging method or species. The analysis method provided is very well applicable to other imaging techniques such as optical imaging of intrinsic signals or fMRI.

\section{ACKNO WLEDGMENT}

This work has been supported by the German Science F oundation (DFG: OB 102/3-1, SE 931/1-1). 


\section{REFERENCES}

Blasdel, G. G. 1992. Differential imaging of ocular dominance and orientation selectivity in monkey striate cortex. J . Neurosci. 12: 3115-3138.

Blasdel, G. G., and Salama, G. 1986. Voltage-sensitive dyes reveal a modular organization in monkey striate cortex. Nature 321: 579585.

Bonhoeffer, T., and Grinvald, A. 1996. Optical imaging based on intrinsic signals: The methodology. In Brain Mapping: The Methods (A. Toga and J . C. Maziotta, Eds.), pp. 55-97. Academic Press, San Diego.

Bygrave, F. L., and Benedetti, A. 1996. What is the concentration of calcium ions in the endoplasmatic reticulum? Cell Calcium 19: 547-551.

Galizia, C. G., J oerges, J ., Küttner, A., Faber, T., and Menzel, R. 1997. A semi-in-vivo preparation for optical recording of the insect brain. J . Neurosci. Methods 76: 61-69.

Galizia, C. G., Nägler, K., Hölldobler, B., and Menzel, R. 1998. Odour coding is bilaterally symmetrical in the antennal lobes of honeybees (Apis mellifera). Eur. J . Neurosci. 10: 2964-2974.

Galizia, C. G., Sachse, S., Rappert, A., and Menzel, R. 1999. The glomerular code for odor representation is species specific in the honeybee Apis mellifera. Nat. Neurosci. 2: 473- 478.

Grinvald, A., Lieke, E., Frostig, R. D., Gilbert, C. D., and Wiesel, T. N. 1986. Functional architecture of cortex revealed by optical imaging of intrinsic signals. Nature 324: 361-364.

J oerges, J ., Küttner, A., Galizia, C. G., and Menzel, R. 1997. Representation of odours and odour mixtures visualized in the honeybee brain. Nature 387: 285-288.

Kao, J . P. Y. 1994. Practical aspects of measuring $\left[\mathrm{Ca}^{2+}\right]$ with fluorescent indicators. Methods Cell Biol. 40: 155.
Kay, S. M. 1993. Fundamentals of Statistical Signal Processing: Estimation Theory. Prentice Hall International, Englewood Cliffs, NJ .

Naraghi, M., and Neher, E. 1997. Linearized buffered $\mathrm{Ca}^{2+}$ diffusion in microdomains and its implications for calculation of $\left[\mathrm{Ca}^{2+}\right]$ at the mouth of a calcium channel. J . Neurosci. 17: 6961- 6973.

Poenie, M. 1992. Measurement of intracellular calcium with fluorescent calcium indicators. Neuromethods 20: 129-174.

Sachse, S., Rappert, A., and Galizia, C. G. 1999. The spatial representation of chemical structures in the antennal lobe of honeybees: Steps towards the olfactory code. Eur. J . Neurosci. 11: 3970-3982.

Schießl, I., Stetter, M., Mayhew, J . E. W., Askew, S., McLoughlin, N., Levitt, J . B., Lund, J . S., and Obermayer, K. 1999. Blind separation of spatial signal patterns from optical imaging records. In Proceedings of the 1st I CA99 Workshop, Aussois, (J .-F. Cardoso, C. J utten, and P. Loubaton, Eds.), Vol. 1, pp. 179-184.

Slayman, C. L., and Moussatos, V. V. 1994. Endosomal accumulation of pH indicator dyes as acetoxymethyl esters. J . Exp. Biol. 196: 419.

Stetter, M., SchießI, I., Otto, T., Sengpiel, F., Hübener, M., Bonhoeffer, T., and Obermayer, K. 2000. Principal component analysis and blind separation of sources for optical imaging of intrinsic signals. Neurol mage 11: 482- 490.

Tsien, R. W., and Tsien, R. Y. 1990. Calcium channels, stores, and oscillations. Annu. Rev. Cell Biol. 6: 715-760.

Tsien, R. Y. 1980. Biochemistry 19: 2396.

Verkhratsky, A., and Kettenmann, K. 1996. Calcium signalling in glial cells. Trends Neurosci. 19: 346-352.

Virgilio, F. D., Steinberg, T. H., and Silverstein, S. C. 1990. Inhibition of fura-2 sequestration and secretion with organic anion transport blockers. Calcium 11: 57.

Xu, T., Naraghi, M., Kang, H., and Neher, E. 1997. Kinetic studies of $\mathrm{Ca}^{2+}$ binding and $\mathrm{Ca}^{2+}$ clearance in the cytosol of adrenal chromaffin cells. Biophys. J . 73: 532-545. 